\title{
Editorial
}

\section{How Fast Can Our Horses Go? Measuring the Quality of Positioning Technologies}

\author{
Anahid Basiri \\ (School of Geographical and Earth Sciences, The University of Glasgow, Glasgow, UK) \\ (E-mail: ana.basiri@glasgow.ac.uk; journal@rin.org.uk)
}

\begin{abstract}
Whether Henry Ford or someone else gave us this famous quote, 'If I had asked people what they wanted, they would have said faster horses', we may agree that it implies there is a limit to what we can expect from the performance of an existing solution. Science and technology always try to push the boundaries and 'improve'; improving the quality of our lives or improving the quality of technologies. We, as researchers in the area of navigation, are no exception; we want to improve the quality of navigation services. And there are many ways to do so, and challenges and limitations to those attempts. Some researchers look to improve the accuracy, the reliability, the integrity through different approaches. Some try to reduce or model noise, some try to minimise human error, and some use novel techniques and algorithms for better prediction. Of course, when 'our horses cannot go any faster' and there is not much space for improvement for a certain technology or service, researchers may come up with a completely new solution, such as an automobile. Almost all new technologies go through the same exploration period; at the beginning, we want to see how and if it works so we try simple tasks, but then we become more ambitious (or greedier!) and so we introduce it to more difficult challenges until it hits the breaking point. At this point, curious researchers and inventors try to push the boundaries and make the technology better, and if improvement is not possible, they build (invent) a new solution. But what is the 'quality' that many of us want to improve? How the quality of a technology or service can be measured in the first place?
\end{abstract}

1. QUALITY OF SERVICE. The International Organisation for Standardisation (ISO) - and many others, including the International Electrotechnical Commission or Open Geospatial Consortium - have defined quality of service (QOS) and enumerated some of the characteristics and aspects of quality. But in general, it is agreed that quality refers to the 'non-functional' aspects of an object, service, technology, dataset, etc. Functional aspects define what a service is supposed to do and non-functional requirements define how a service is supposed to be.

Do you remember the first time you held a mobile phone? It was certainly an exciting moment to see a smaller phone with no coiled cords that could ring and connect us, 
regardless of our location. Many of us conducted the same experiment, calling home to see if it actually worked! Then we walked a little further to see how far we could go before losing the connection. At that point, the only feature that was tested was the 'functionality' of the new mobile phone. Nowadays, when we want to buy a new mobile phone, we do not even think about 'functionality'; it should work! There are other factors and features to consider; the battery consumption, quality of the camera, cost, size etc. In fact, the functionality of the phone is no longer considered as a factor, as we assume it works and we simply focus on the non-functional aspects. These non-functional aspects are now 'quality' aspects.

2. QUALITY ASPECTS OF NAVIGATION AND POSITIONING. In our area of interest, i.e. navigation, we can define the key functional requirement of a navigation service as delivering the user's position or navigational instruction. On the other hand, providing the position in real time, or in a privacy-preserving mode, can be considered a non-functional requirement of the service. One can argue that for some services, realtime delivery is the key, without which the service is useless. While that is true, there is a minimum requirement for some of the non-functional aspects, depending on the context or applications. For example, in order to expect users to use an in-car navigation device, we may expect a minimum level of accuracy or reliability; otherwise, the devices may be thought to be 'not working'. The boundary between functional and non-functional requirements may blur for some applications or context. However, we have created a list of some aspects that could be considered as non-functional attributes, in general. They include:

- (Positional, Temporal, Semantic) accuracy

- Reliability

- Resilience

- Compliance

- Stability

- Consistency

- Scalability

- User-friendliness

- Response time

- Portability

- Initial cost

- Life-cycle cost

- Durability

- Dependency (on other parties or systems)

- Integrability (and interoperability)

- Coverage (and reach)

- Accessibility

- Privacy

- Security

- Recoverability

- Safety

- Trustworthiness

- Transparency

- Interoperability

- Reproducibility (and testability) 
Here, we can add a few more aspects that seem to be essential, as the role of data-centric systems and intelligent technologies have become more and more important. They include ethics, fairness, and inclusivity of the technologies and services.

Some of these are well researched in our research area, i.e. navigation. For example, many have tried to improve the accuracy of the position solution. Accuracy (defined as the closeness of the measurements to a specific value (e.g. true location of the user)) is certainly one of the most important aspects of any location-based service, including navigation. Therefore, improving the accuracy of any positioning technology has been the topic of research for quite a long time, and so we have seen a huge improvement in terms of achievable accuracy from almost all positioning technologies. For example, with more positioning signals around, more Global Navigation Satellite System (GNSS) satellites on sky broadcasting multi-frequencies, and a better understanding of the environment using three-dimensional maps have enabled researchers to remove, minimise or model many sources of errors and uncertainties. These have resulted in significant improvement in the achievable accuracy of the instruments. In this issue of the Journal of Navigation, Basile et al. (2020), Jiao et al. (2020), Macho et al. (2020) and Zhao et al. (2020) specifically look at the accuracy aspect of positioning solutions using GNSS signals using different approaches. They include using multi-frequency ionosphere-free data to improve the observability and ambiguities, multi-station tropospheric models that can factor in the impacts of height.

Some of these 'non-functional aspects can be considered as a subset, dependent on or highly correlated to some of the other aspects. For example, the cost may increase or, in fact, can be a function of accuracy for many sensors. If the correlation is negative, then there is a trade-off between some of the quality aspects. For example, if we integrate additional information about the environment, e.g. 3D maps or map-matching, or use other sensors and signals in order to provide more reliable positioning, the system may not be as standalone as it used to be, though it may provide a higher level of accuracy or reliability. This can be an issue in some contexts and applications and so one may need to judge which quality aspect is important and how much value it offers. In this issue, Liu et al. (2020) looks at the balance between cost and accuracy, and proposes a map-matching-based solution to provide a low-cost positioning service for vehicle navigation.

While finding the balance may require the system to be prepared for some levels of compromises, for some applications there is a priority list of and clear regulations on what aspects can or cannot be compromised. For example, civil aviation seems to be very well regulated in terms of integrity, reliability and accuracy, as safety is the top priority. Researchers with interests in maritime have also investigated these aspects using different approaches. In this issue of the journal, Alizadeh et al. (2020), Castells-Sanabra et al. (2020) and Fiskin et al. (2020) propose and implement ways to minimise risks of collision or accidents. Fiskin et al. (2020) used data-mining techniques to understand the factors contributing to accidents involving tugboats. Castells-Sanabra et al. (2020) designed and developed a simulator that can be used by port authorities to enable them to prevent the effects of ship erosion on harbour infrastructures.

All in all, what we do as researchers is push boundaries and try to improve one or more aspects of quality, and when our horses cannot go any faster, we may invent or repurpose technologies. I look forward to seeing how positioning and navigation will improve even further using new signals such as $5 \mathrm{G}$ and $6 \mathrm{G}$, how quantum technology will help our mobile phone to localise more accurately, and how autonomous vehicles will improve in terms of safety and reliability. 


\section{REFERENCES}

Alizadeh, D., Alesheikh, A. and Sharif, M. (2020). Vessel trajectory prediction using historical automatic identification system data. Journal of Navigation, 1-19. doi:10.1017/S0373463320000442.

Basile, F., Moore, T., Hill, C. and McGraw, G. (2020). GPS and galileo triple-carrier ionosphere-free combinations for improved convergence in precise point positioning. Journal of Navigation, 1-19. doi: $10.1017 / \mathrm{S} 0373463320000454$

Castells-Sanabra, M., Mujal-Colilles, A., LLull, T., Moncunill, J., Martínez de Osés, F. and Gironella, X. (2020). Alternative manoeuvres to reduce ship scour. Journal of Navigation, 1-18. doi:10.1017/S0373463320000399.

Fiskin, R., Cakir, E. and Sevgili, C. (2020). Decision tree and logistic regression analysis to explore factors contributing to harbour tugboat accidents. Journal of Navigation, 1-26. doi:10.1017/S0373463320000363.

Jiao, C., Wang, X., Wang, D., Li, Q., Zhang, J. and Cai, Y. (2020). An adaptive vector tracking scheme for high-orbit degraded GNSS signal. Journal of Navigation, 1-20. doi:10.1017/S0373463320000387.

Liu, F., Liu, Y., Nie, Z. and Gao, Y. (2020). Precise single-frequency positioning using low-cost receiver with the aid of lane-level map matching for land vehicle navigation. Journal of Navigation, 1-14. doi:10.1017/S0373463320000375.

Macho, E., Pamboukian, S. and Correia, E. (2020). Geolocal - a new system for geo-referencing: analysis of base distribution. Journal of Navigation, 1-13. doi:10.1017/S0373463320000466.

Zhao, Q., Pan, S., Gao, C., Qiao, L., Gao, W., Zhang, R. and Liu, G. (2020). A multi-station troposphere modelling method based on error compensation considering the influence of height factor. Journal of Navigation, 1-22. doi:10.1017/S037346332000034X. 